\title{
ForestTime: una aplicación móvil para el estudio de tiempos de trabajo de maquinaria forestal a través de teléfonos inteligentes
}

\author{
ForestTime: a mobile application to forest machinery work study through smartphones \\ Eduardo Acuña a*, Pablo Mena ${ }^{\text {a }}$, Claudio Torres ${ }^{\text {a }}$, Jorge Cancino ${ }^{\text {a }}$ \\ *Autor de correspondencia: a Universidad de Concepción, Facultad de Ciencias Forestales, \\ Victoria 631, ciudad Universitaria, Concepción, Chile, edacuna@udec.cl
}

\begin{abstract}
SUMMARY
Today's smartphones and tablets offer computing capabilities and storage attractiveness, which allow a variety of mobile applications with great functionality. Integrating new interfaces opens up new opportunities for new applications. These applications require storage and processing of data on smartphones. This article presents the design and implementation of an application, which aims at keeping records of actual working time of forestry machinery. The application was programmed in Eclipse under the application programming interface for Android, following the methodology proposed by IUFRO. The application allows users to measure performance over time and forestry machinery wishing to incorporate into their records, and also enables users to manipulate databases, performing statistical analyses after the requirements of stakeholders. The application on Android smartphones shows that it can bring the benefits Foresters through the digital recording of data for a timely and effective forest management.
\end{abstract}

Key words: smartphone application, forestry, forest work study, machinery, field work, android.

\section{RESUMEN}

Los teléfonos inteligentes y tabletas de hoy ofrecen capacidades de cómputo y almacenamiento atractivos, los cuales permiten una gran variedad de aplicaciones móviles con una gran funcionalidad. La integración de nuevas interfaces abre oportunidades para las nuevas aplicaciones. Estas aplicaciones requieren almacenamiento y procesamiento de datos en los teléfonos inteligentes. En este artículo se presenta el diseño e implementación de una aplicación, que tiene por finalidad llevar el registro del tiempo de trabajo real de las maquinarias. La aplicación "ForestTime" se programó en Eclipse bajo la interface de programación de aplicaciones de Android y siguiendo la metodología propuesta por la IUFRO. La aplicación permite a los usuarios medir el tiempo y rendimiento de las maquinarias forestales que deseen incorporar dentro de sus registros, asimismo permite a los usuarios exportar la base de datos generada y realizar posteriores análisis estadísticos acordes con los requerimientos de los interesados. La aplicación desarrollada en Android demuestra que los teléfonos inteligentes pueden traer beneficios a los ingenieros forestales a través del registro digital de datos para una gestión forestal oportuna y eficaz.

Palabras clave: aplicación para teléfono inteligente, silvicultura, estudio de trabajo forestal, maquinaria forestal, trabajo de campo, Android.

\section{INTRODUCCIÓN}

La economía de Chile es muy dependiente de la industria primaria, siendo la actividad forestal uno de los sectores más significativos. Un reto importante para el sector está radicado en el cultivo y cosecha de árboles en terrenos de topografía difícil. Las empresas forestales durante las décadas de los 1980-1990 compraron tierras más baratas para plantar $\mathrm{y}$, producto de ello, establecieron grandes superficies de bosques en sitios con pendientes pronunciadas. Para cosechar estos sitios se requieren soluciones de forma segura y costoefectiva para que la industria mantenga su competitividad.

El crecimiento del sector forestal ha obligado a la incorporación de nuevas y más eficientes tecnologías en las faenas silvícolas. Así, las empresas de servicios han realizado cuantiosas inversiones en el logro de la mecanización de los procesos. La cosecha forestal ha tenido un desarrollo extraordinario en los últimos años, gracias a la incorporación de maquinarias con tecnología de vanguardia. Esta no sólo ha permitido aumentar la productividad sino también la seguridad en las operaciones (Pasicott y Murphy 2013). Las principales innovaciones en faenas de cosecha, están focalizadas en el volteo, transporte primario, desrame y procesamiento de los árboles, con la incorporación de harvesters, feller bunchers y skidders (Lignum 2013, Pasicott y Murphy 2013).

En general, las operaciones forestales mecanizadas se subcontratan a empresas de servicios (Durán-Palma y López 2009), las que se encuentran insertas en la mayoría de las etapas del ciclo productivo. Ello hace necesario evaluar la productividad de los equipos utilizados en los distintos sistemas de aprovechamiento forestal. 
Para evaluar el rendimiento de las actividades silvícolas, los silvicultores llevan siempre con ellos a terreno cuadernos y lápices para tomar notas, GPS, instrumentos de medición y mapas para hacer seguimientos de las distintas paradas de la maquinaria, siendo la lista de implementos más larga cuanta más información se desee obtener o documentar mejor el trabajo de campo. Sin embargo, como el número de herramientas aumenta también lo hacen los tipos de datos recogidos y esto plantea problemas en sí mismo. Primero, es un inconveniente llevar un gran número de herramientas a campo. En segundo lugar, la tarea posterior de relacionar datos obtenidos de los diferentes dispositivos puede ser un reto y la dificultad aumenta con la variedad de datos.

Las nuevas tecnologías móviles podrían hacer que el procesamiento in situ de datos e información oportuna proporcionen soluciones accesibles para la gestión forestal. Es así que para resolver el problema de evaluar el tiempo y rendimiento de maquinaria forestal, la literatura registra dos diseños computacionales para controlar diferentes actividades desarrolladas en los aprovechamientos forestales (motosierrista, apilador, cosechadora, autocargador, skidder, tractor forestal, cable y tracción animal). Para ello, los registros de datos en campo se realizan sobre libretas electrónicas, handheld o personal digital assistant (PDA). El primero de ellos es SIWORK 3, versión 1.1 sobre un antiguo computador denominado Husky Hunter (Rolew 1988) y, uno más reciente, el programa KRONOS sobre la handheld de marca PSION workabout (Ambrosio y Tolosana 2007). Ambas soluciones computacionales programadas en lenguajes de uso exclusivo por estos aparatos electrónicos y por lo tanto poco masivos.

Por otro lado, los teléfonos inteligentes o tabletas ofrecen ayuda inmediata a los problemas mencionados anteriormente, dada la gran cantidad de funciones con los que están equipados. Estos, como los equipos iPhone, iPad y aparatos con sistema operativo Android (Menard et al. 2011), ofrecen amplias capacidades de almacenamiento en el rango de 8 a 64 gigabyte, además de conectividad mediante redes Wi-Fi o 3G/4G (Wong 2011). Pueden almacenar cientos, si no miles de archivos a la vez, los que pueden posteriormente ser transferidos a través de Internet a una ubicación remota para el almacenamiento permanente.

Los teléfonos inteligentes como las tabletas vienen con una serie de funciones deseables, tales como teclado/teclado virtual, cámara, grabadora, brújula digital, GPS y acelerómetro (Nelson 2012). Estos, por lo tanto, pueden tomar el lugar de una variedad de dispositivos y proporcionar las funciones necesarias para la toma de datos de campo.

La informática móvil ha influido en la vida cotidiana hasta tal punto que gran parte de la población se encuentra en línea en cualquier momento del día y en cualquier lugar. Ahora es posible usar aplicaciones de software sofisticadas (applications) en la mano (Monsen 2012), que hace pocos años estaban limitadas a equipos de escritorio. La movilidad ya no se espera que se limite a encender en terreno un equipo, sino más bien la simple apertura de un dispositivo de mano que está siempre encendido y siempre conectado.

Las referencias bibliográficas de aplicaciones en tabletas y teléfonos inteligentes que ayuden en la gestión forestal son limitadas en la literatura académica. Algunos ejemplos son los de Dianyuan y Hui (2011) quienes estudian la medición de árbol en pie basado en el procesamiento de imágenes; Dianyuan y Chengduan (2011) e Itoh et al. (2010) desarrollan aplicaciones para medir la altura de los árboles, los primeros a través de imágenes y los segundos aprovechando el acelerómetro de los teléfonos inteligentes; finalmente, García (2011) desarrolla una aplicación para el sistema operativo móvil Android para el guiado de bomberos en los incendios forestales, capaz de controlar en el mapa la posición de los bomberos en un incendio.

El estudio del tiempo y el rendimiento de las maquinarias forestales es un indicador importante en la gestión forestal, dado que proporciona la capacidad de tomar decisiones tanto a nivel económico como logístico. La obtención de estos indicadores en terreno permite aumentar la eficacia del proceso productivo.

La falta de uniformidad de los conceptos de tiempo en estudios de tiempo y rendimiento fue identificada por los autores de este artículo, y es considerada como el obstáculo más importante cuando se trata de hacer comparaciones nacionales o internacionales de informes sobre este tema. Por ello, el objetivo de este artículo es el diseño, programación y evaluación de una aplicación para dispositivos móviles con sistema operativo Google Android, para el estudio de tiempo y rendimiento de maquinaria forestal, siguiendo la metodología propuesta por la International Union of Forest Research Organizations (IUFRO) (Björheden y Thompson 1995). La creación y uso de esta aplicación favorecerá a los profesionales forestales y a distribuidores de maquinaria forestal en la toma de datos de campo, así como en la gestión de estos, en estudios de tiempo y rendimiento para la importación y adquisición informada del desempeño de equipos forestales.

\section{MÉTODOS}

Metodología de programación. El desarrollo de la aplicación "ForestTime” utilizó un modelo de ciclo de vida de software definido por Boehm (1988), el cual es conocido como desarrollo en espiral. Boehm propone cuatro actividades a repetir iterativamente hasta concluir con la aplicación; estas son: determinación de objetivos, análisis de riesgo, planificación y desarrollo (verificación y validación). El programa "ForestTime" es una aplicación de Android, que tiene por finalidad llevar el registro del tiempo de trabajo real de las máquinas.

La aplicación se programó en el entorno de desarrollo Eclipse, bajo la interfaz de programación de aplicaciones (IPA) o API (del inglés application programming interface) de Android. Se eligió esta plataforma para programar puesto que es la más masiva en el país. El procedimiento 
de análisis seguido para el desarrollo de este programa es una base lógica y búsqueda de información pertinente al desarrollo de aplicaciones en Android. Ejemplo de ello fue la búsqueda de información necesaria para trabajar con cronómetros en Android, la manera de iniciarlos, de detenerlos y volver al inicio usando botones. Esto es una metodología de tiempo cero, es decir, que cada vez que se intenta medir una actividad de la máquina, el tiempo total sigue andando, pero se detiene el sub-cronómetro que cuantifica el tiempo de la actividad en sí para medir otra; así, cada vez que se cambia de actividad, el cronómetro avisa y calcula la diferencia entre el tiempo anterior y el actual para llevar un conteo real del tiempo ocupado.

Esquema del funcionamiento. El funcionamiento de la aplicación se puede descomponer en tres fases: a) selección e ingreso de maquinaria, donde el usuario puede seleccionar o incorporar una nueva maquinaria a evaluar; b) medición del intervalo de tiempo, fase en que se crea un registro en la base de datos con información del tiempo transcurrido entre cada actividad realizada por la máquina; y c) cambio de actividad, fase en que se puede realizar cambio de actividad, según estipula la metodología propuesta por IUFRO para este tipo de estudios (Björheden y Thompson 1995).

La jornada de medición finaliza determinando tres indicadores de rendimiento: la disponibilidad mecánica (MA), la utilización de la máquina (MU) y la capacidad de utilización de la máquina (CU). De acuerdo a Van Daele (2000), la disponibilidad mecánica es la porción de tiempo en el lugar de trabajo en la cual un equipo se encuentra mecánicamente disponible y es capaz de hacer el trabajo productivo, la utilización de la máquina es el porcentaje de tiempo que la máquina -en el lugar de trabajo- se encuentra realizando el trabajo para la que fue diseñada y, la capacidad de utilización mide en qué grado el tiempo total es utilizado por la máquina para realizar el trabajo (el tiempo de trabajo, en este caso, incluye todos los retrasos, tiempos de apoyo y los tiempos productivos). Esta última no es una medida de la eficiencia de la máquina, sino una medición de las horas de trabajo regulares de la operación contra el tiempo calendárico total disponible. Así los indicadores de rendimiento son:

Disponibilidad mecánica (mechanical availability) (MA), ecuación [1]:

$\mathrm{MA}(\%)=\frac{\mathrm{WP}-\mathrm{ST}}{\mathrm{WP}} \times 100$

Utilización de la máquina (machine utilisation) $(M U)$, ecuación [2]:

$\mathrm{MU}(\%)=\frac{\mathrm{PW}}{\mathrm{WP}} \times 100$

Capacidad de utilización (capacity utilisation) (CU), ecuación [3]:

$\mathrm{CU}(\%)=\frac{\mathrm{WP}}{T T} \times 100$
Donde:

$W P=$ Tiempo de trabajo (workplace time).

$S T=$ Tiempo de servicio (service time).

$P W=$ Tiempo de trabajo productivo (productive work time). $T T=$ Tiempo total (total time).

Evaluación de ForestTime en cosecha forestal. El área de estudio fue un conjunto de rodales de Eucalyptus globulus Labill. establecidos los años 2002 y 2003, ubicados en el predio Las Mellizas cercano a la ciudad de Angol, Chile. El predio posee una topografía de lomajes con una pendiente promedio del $14 \%$, un máximo de $42 \%$ y un mínimo de $3 \%$. El inventario precosecha proyectó una densidad de 1.085 árboles ha $^{-1}$, un volumen de 148,06 m ha $^{-1} \mathrm{y}$ un diámetro medio cuadrático de $23,6 \mathrm{~cm}$. Esas variables de estado de rodal sugieren una cosecha para pulpa debido al pequeño tamaño de los árboles $\left(0,14 \mathrm{~m}^{3}\right.$ árbol $\left.{ }^{-1}\right)$ (cuadro 1).

El sistema de aprovechamiento fue de árbol entero (whole tree system) e incluyó un cosechador de orugas (Tigercat Shovel Logger LS855C), un skidder de garra (Tigercat Grapple skidder 625) y un skidder de huinche (Tigercat cable skidder 604C) para el transporte primario y un procesador-trozador (Timberpro 630) (figura 1). El cosechador de orugas derribó los árboles y los apiló en gavillas; luego los skidders de garra y de huinche trasladaron las gavillas a la cancha de aprovechamiento en donde estaba ubicado el procesador Timberpro.

Los tiempos de trabajo se registraron para el sistema de aprovechamiento completo desde el cosechador hasta el procesador. Para ello, cuatro operarios, uno por cada máqui-

Cuadro 1. Tabla de rodal de precosecha. Preharvest stand table.

\begin{tabular}{ccccc}
\hline $\begin{array}{c}\text { Clase DAP a } \\
(\mathrm{cm})\end{array}$ & $\begin{array}{c}\text { Densidad } \\
\left(\text { árboles ha }^{-1}\right)\end{array}$ & $\begin{array}{c}\text { Altura } \\
(\mathrm{m})\end{array}$ & $\begin{array}{c}\text { Área basal } \\
\left(\mathrm{m}^{2} \mathrm{ha}^{-1}\right)\end{array}$ & $\begin{array}{r}\text { Volumen } \\
\left(\mathrm{m}^{3} \mathrm{ha}^{-1}\right)\end{array}$ \\
\hline 8 & 45 & 11,0 & 0,23 & 0,80 \\
10 & 65 & 12,5 & 0,51 & 2,13 \\
12 & 145 & 13,8 & 1,64 & 7,69 \\
14 & 210 & 15,1 & 3,23 & 16,68 \\
16 & 175 & 16,2 & 3,52 & 19,68 \\
18 & 180 & 17,4 & 4,58 & 27,47 \\
20 & 70 & 18,4 & 2,20 & 14,02 \\
22 & 100 & 19,4 & 3,80 & 25,61 \\
24 & 70 & 20,4 & 3,17 & 22,43 \\
26 & 10 & 21,3 & 0,53 & 3,94 \\
28 & 10 & 22,3 & 0,62 & 4,76 \\
30 & 5 & 23,1 & 0,35 & 2,84 \\
32 & 5 & 24,0 & 0,40 & 3,36 \\
\hline
\end{tabular}

${ }^{a}$ DAP: diámetro de fuste a la altura del pecho, i.e. a 1,3 m desde el nivel del suelo. 
Volteo

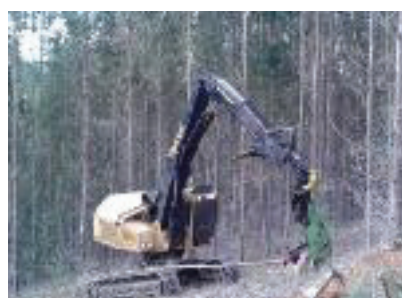

Tigercat Shovel Logger LS855C

Trasporte primario

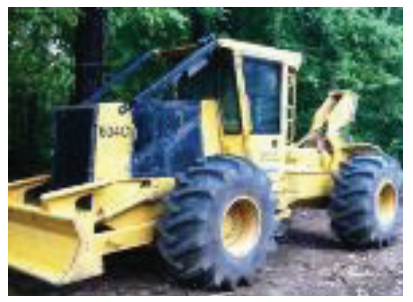

Tigercat Cable Skidder 604C
Transporte primario

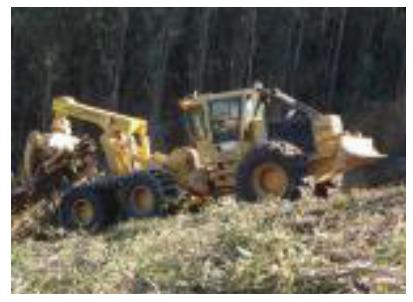

Tigercat Grapple Skidder 615

Trozado

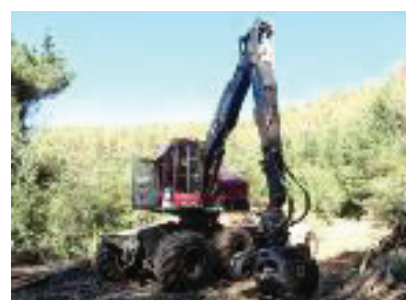

Timberpro 630
Figura 1. Sistema de cosecha de la biomasa en el área de estudio. Biomass harvest system in the study area.

na, observaban diariamente las actividades de las máquinas y registraban en la aplicación ForestTime los tiempos empleados por cada una de ellas, de acuerdo a la metodología propuesta por Björheden y Thompson (1995) (figura 2). En este artículo se ilustran solo los registros del skidder huinche Tigercat 604C. Los tiempos de ciclo de este skidder se dividieron en seis actividades: estrobado, viaje cargado, desestrobado, viaje vacío, tiempo perdido y ordenamiento.

\section{RESULTADOS}

"ForestTime" consta de seis módulos principales los cuales son: ingresar datos maquinaria, eliminar datos maquinaria, seleccionar datos maquinaria, ingresar datos tiempos, mostrar datos resultantes.

- Ingresar datos maquinaria: se ha creado una base de datos para que se puedan ingresar las maquinarias a las cuales se les medirá el tiempo y los indicadores de rendimientos señalados con anterioridad. Con esto se puede validar que no se ingrese una misma maquinaria más de una vez, además de poder mostrar en un módulo de selección cada una de las maquinarias ingresada para poder finalmente elegir una de ellas y medir su rendimiento.

- Eliminación datos maquinaria: este módulo permite eliminar algún dato mal ingresado de las maquinarias. Este módulo, por tanto, solo elimina las maquinarias de su base de datos, pero no elimina el registro de la eficiencia de esta maquinaria en la base de datos de tiempos.

- Seleccionar datos maquinaria: este módulo permite elegir de una lista de maquinarias ya ingresadas por el usuario, la máquina a la que se la va a medir tiempo y eficiencia en las actividades propuestas por el programa (figura 3).

- Ingresar datos tiempos: un vez que se ha elegido la maquinaria en el módulo de selección, se podrá medir su tiempo (figura 4A) y además ingresar en la base de datos de tiempos. Ingresa el tiempo total en cada actividad que se ha realizado; en las actividades que no se han llevado a cabo, el tiempo será 0 .

En la determinación del tiempo se utiliza un cronómetro de tiempo total, en donde, cada vez que se cambia de actividad, se despliega el tiempo parcial y se determina la diferencia de tiempo entre de la actividad anterior y la ac-

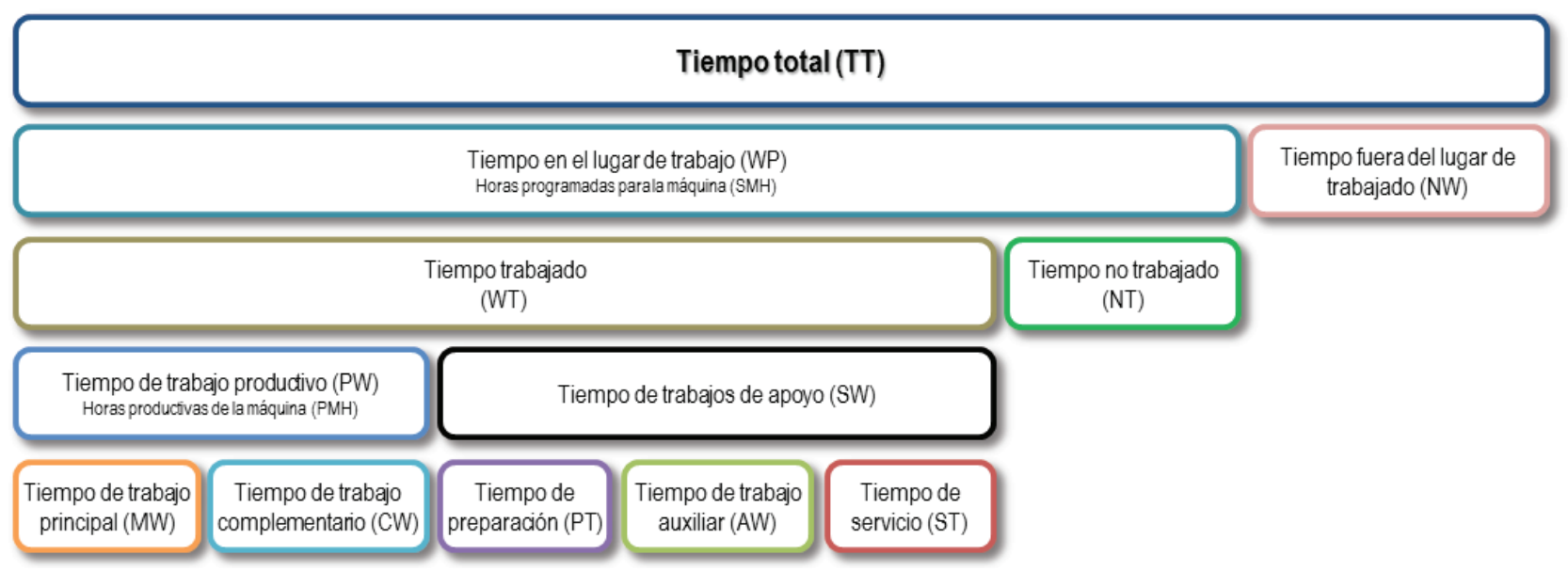

Figura 2. Componentes básicos de la distribución de tiempo. Basic time components breakdown. 


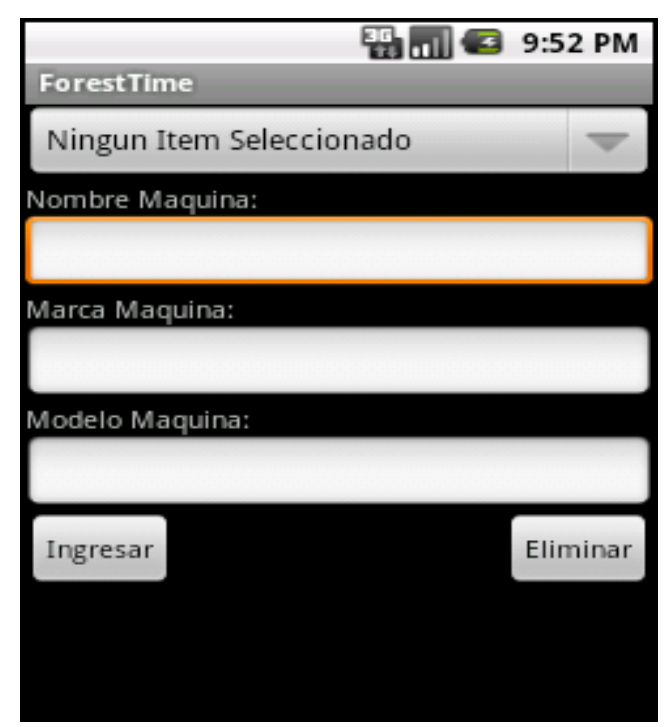

Figura 3. Ventana de selección e ingreso de maquinaria. Display of selection and machinery entry.

tual, que corresponde al tiempo real ocupado (figura 4A). $\mathrm{Al}$ presionar un botón de actividad por primera vez, también se despliegan los botones "Stop" y "Reset".

Además de estos tres módulos básicos, el programa tiene la opción de salir de la aplicación en todo momento, así como también mostrar en pantalla la información de cómo usar el programa y otra información relevante para el usuario:

- Mostrar datos resultantes: cuando se presiona el botón "Stop", el conteo de segundos del cronómetro se detiene y se despliega el botón "Resume", que sintetiza el tiempo de la actividad. El botón "Reset" vuelve a cero el cronómetro (figura 4B). Después de medir el tiempo de cada actividad el programa está dotado para mostrar los datos resultantes y cálculos sobre la eficiencia de la maquinaria, los que determinarán qué tan eficiente fue el trabajo (figura 5).

Al momento de presionar "Stop", también se desplegará el botón "Ingresar a la base de datos". Con ello se ingresan los datos a ésta y se cambia de pantalla mostrando los tiempos en segundos, minutos y horas ocupados por cada actividad, además de los cálculos sobre la eficiencia de la maquinaria (ecuaciones 1 a 3). Las bases de datos de maquinarias y tiempos quedan almacenadas dentro de la memoria del celular y pueden ser consultadas para realizar cálculos posteriores de acuerdo a las necesidades de información del usuario. Para ingresar a los archivos se debe ingresar a la carpeta /data/data/forest.time, del sistema.

Registro de campo. En el estudio se controlaron 82 horas programadas en ocho días de trabajo del sistema de cosecha de árbol completo (cuadro 2). El trabajo fue realizado todos los días por el mismo operario y no se presentaron disturbios producto de inclemencias del tiempo.

Los tiempos de retraso para el skidder huinche fueron el $22,65 \%$, en donde las alteraciones al trabajo, interferencias y tiempo para comida fueron del 3,6 \%, 6,4 \% y 12,4 \% del tiempo de operación total, respectivamente. El tiempo efectivo el transporte primario fue del 57,9 \% del tiempo total de trabajo (figura 6). Los retrasos en la operación fueron causados por visitas de la empresa mandante y supervisores en el caso de las alteraciones y el de la necesidad de trabajos de limpieza de la cancha de trozado, en el caso de las interferencias.
A)

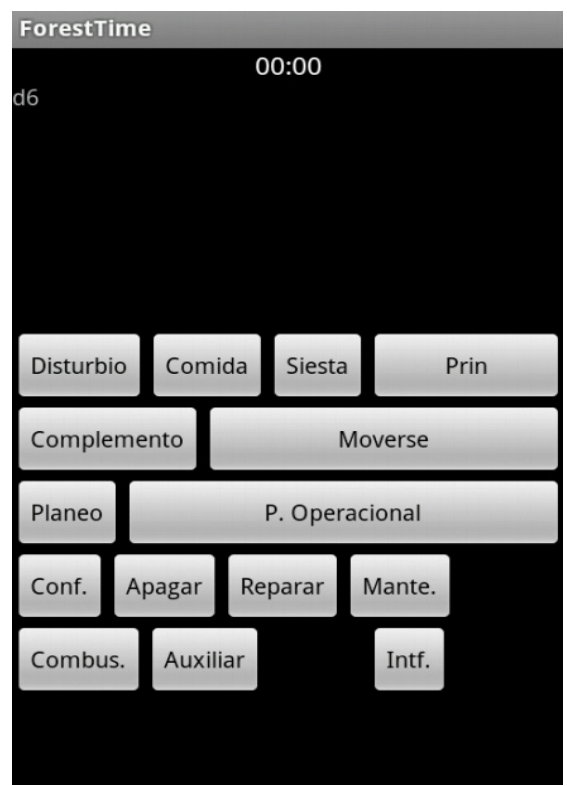

B)

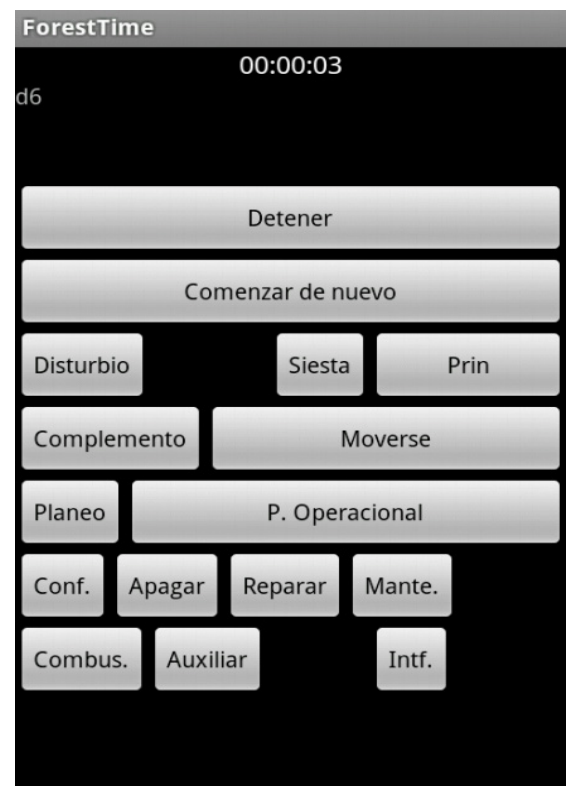

Figura 4. A) Ventana de Selección de actividades. B) Ventana de selección de una nueva actividad.

A) Display of activities selection. B) Display for selecting a new activity. 


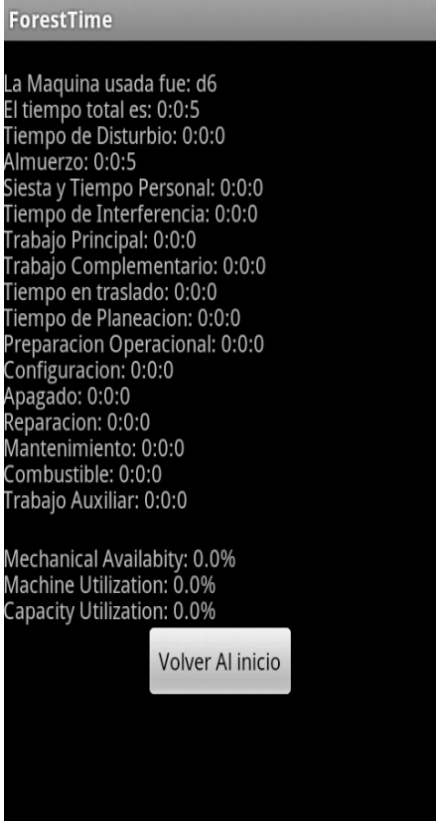

Figura 5. Ventana de resultados de estudio de tiempos. Display of time study outcome.

Las actividades de operación del transporte primario, viajes cargado y vacíos del skidder ocuparon la mayor porción del tiempo principal de trabajo $(25,3 \%$ y $18,3 \%$, respectivamente). Las demoras (retrasos operacionales y otros) comprendieron el 19,3\% del total de las operaciones. Los retrasos fueron causados principalmente por reparaciones (6,6 \%) producto del corte del cable de arrastre, complementarios producto del ordenamiento de madera en la cancha de trozado (5,9\%), traslado por reubicación de la máquina (2,6 \%) y por mantención (2,6 \%). Combustible y viajes fueron otros factores de los retrasos (figura 6).

De acuerdo a los indicadores de rendimiento evaluados, el skidder huinche Tigercat 604C tuvo una disponi- bilidad mecánica de un 86,1 \%, una utilización de la máquina de $82,7 \%$ en labores propias de transporte primario (madereo) y, una capacidad de utilización de 77,2%.

\section{DISCUSIÓN}

Las aplicaciones forestales disponibles para los teléfonos inteligentes aún son escasas y la mayoría de ellas no se encuentran registradas en publicaciones científicas. Entre las publicadas se encuentran las de Itoh et al. (2010) quienes desarrollan un software para medir altura de árboles con la ayuda del acelerómetro que traen los teléfonos inteligentes; otra de Dianyuan y Chengduan (2011) y Dianyuan y Hui (2011) -buscando el mismo objetivo- lo hace a través del procesamiento de imágenes integradas a los teléfonos móviles; la de García (2011), quien desarrolló una aplicación de guiado para bomberos en incendios forestales, es capaz de controlar en el mapa la posición de los bomberos en un incendio, ver sus datos y escribir mensajes; y la de Jeong et al. (2011), quienes desarrollan una aplicación para la gestión de inventarios forestales.

Como se puede ver, el desarrollo de aplicaciones para el manejo silvícola es aún pequeño. Esto puede deberse a que el mercado de la silvicultura o sector forestal nacional es limitado en términos de ventas potenciales de este tipo de aplicaciones, cuando se le compara con el mercado de consumo de juegos y aplicaciones de entretenimiento. Con la expectativa adicional de "libre" para muchos usuarios, se pueden generar aplicaciones como vehículo de publicidad, generando altos rendimientos sobre la inversión. Muchas de las versiones de aplicaciones libres o "versiones light" por lo general utilizan baners de publicidad como una manera de generar ingresos. Esto podría obstaculizar el espacio real de la pantalla ya limitado. Sin embargo, los modelos de negocios que involucran el desarrollo de aplicaciones gratuitas o de bajo costo que sirven como herramientas de marketing para las industrias basadas en los servicios

Cuadro 2. Registro de tiempos de skidder huinche Tigercat 604C. Time register of Tigercat $604 \mathrm{C}$ cable skidder.

\begin{tabular}{|c|c|c|c|c|c|c|c|c|c|c|c|c|}
\hline \multirow{2}{*}{ Fecha } & \multicolumn{11}{|c|}{ Componentes de tiempo (hora:minuto:segundo) } & \multirow{2}{*}{$\begin{array}{c}\text { Tiempo } \\
\text { total }\end{array}$} \\
\hline & CW & DT & IT & ME & MT & MW & $\mathrm{RF}$ & RL & $\mathrm{RP}$ & RT & TR & \\
\hline $14-05-2013$ & $0: 46: 47$ & $0: 30: 59$ & $0: 19: 38$ & 1:15:27 & $0: 28: 24$ & 6:17:05 & 0:07:34 & 0:04:58 & $0: 10: 29$ & - & - & 10:01:21 \\
\hline $15-05-2013$ & 1:00:27 & 0:20:00 & - & 1:45:01 & $0: 12: 17$ & 5:24:11 & 0:06:29 & - & - & $1: 25: 54$ & - & 10:14:19 \\
\hline $16-05-2013$ & 0:11:31 & 0:00:40 & $0: 41: 13$ & 1:00:00 & 0:01:29 & 5:12:45 & $0: 34: 47$ & 0:16:58 & - & 2:29:00 & $0: 07: 21$ & $10: 35: 44$ \\
\hline $17-05-2013$ & 0:09:21 & $0: 20: 46$ & 0:46:50 & 1:04:50 & $0: 45: 32$ & 7:09:27 & - & 1:15:39 & - & 0:02:40 & - & 11:35:05 \\
\hline $18-05-2013$ & 1:08:50 & 0:21:04 & $1: 15: 25$ & $1: 22: 20$ & 0:13:57 & 4:52:03 & - & 0:19:11 & - & 0:04:48 & - & 9:37:38 \\
\hline 22-05-2013 & 0:49:05 & $0: 34: 40$ & 1:02:03 & 1:07:46 & 0:02:04 & 6:12:46 & 0:07:21 & 0:05:19 & - & - & - & 10:01:04 \\
\hline 23-05-2013 & 0:06:30 & $0: 46: 24$ & 0:35:04 & 1:19:30 & $0: 22: 31$ & $5: 37: 37$ & 0:07:57 & 0:07:46 & - & 0:57:01 & - & 10:00:20 \\
\hline $24-05-2013$ & $0: 37: 06$ & 0:05:03 & $0: 34: 45$ & $1: 14: 25$ & 0:04:07 & $6: 45: 04$ & 0:07:05 & - & - & $0: 27: 41$ & - & 9:55:16 \\
\hline Total & 4:49:37 & 2:59:36 & $5: 14: 58$ & 10:09:19 & $2: 10: 21$ & $47: 30: 58$ & 1:11:13 & 2:09:51 & $0: 10: 29$ & $5: 27: 04$ & $0: 07: 21$ & 82:00:47 \\
\hline
\end{tabular}




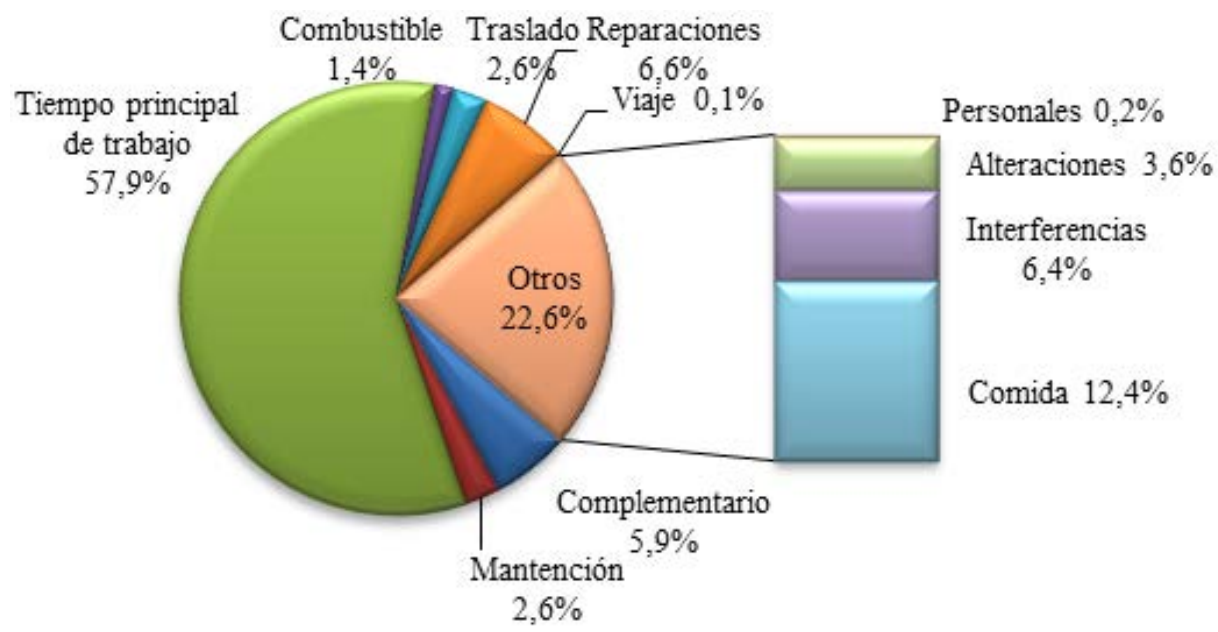

Figura 6. Porcentaje de tiempos empleados por el skidder huinche. Time used for cable skidder as a percent of total time.

relacionados con la silvicultura podría llegar a ser viable (i.e. maquinarias, productos químicos, servicios forestales).

La aplicación que presenta este artículo, denominada ForestTime y destinada al estudio de tiempos y rendimientos en faenas de cosecha forestal, fue probada con éxito en una faena de este tipo. En terreno, cada uno de cuatro encuestadores, uno por cada máquina en evaluación, utilizaron teléfonos inteligentes con ForestTime para realizar el seguimiento de las actividades al mismo tiempo. Las principales dificultades encontradas fueron las relacionadas a la inducción de los encuestadores, quienes debían tener muy claro el significado de cada uno de los componentes de tiempo. A pesar de ello, existió la necesidad de anotar observaciones en una hoja aparte cuando existían dudas; también se registraron las distancias de transporte primario y las pendientes de los viajes cargados y vacíos. La aplicación logró en estos casos aliviar la carga de los encuestadores para sus observaciones ya que la medición de los tiempos se realiza en forma automática apretando el botón de cada actividad.

La evaluación tradicional de las maquinarias forestales, que se realiza solo cuando se importa una nueva máquina, $\mathrm{y}$ que se hace principalmente a través de la digitación o ingreso manual de datos, podría hacerse más habitual mediante el uso de dispositivos móviles incorporándolos en las máquinas, de tal manera que el operario sea el que incorpore los registros de datos. Así, se obtendría una amplia gama de registros por especie, clase de pendiente, clima, operario, etc., la cual podría ser gestionada por la gerencia de las empresas de servicios para implementar mejoras que conduzcan a reducción de costos y aumento de la productividad.

La evaluación de ForestTime en terreno permitió identificar deficiencias que, una vez corregidas, permitirán contar con una herramienta versátil y cómoda para estudios de tiempo. Entre otras es necesario: i) incorporar observaciones (texto) -en las etapas de tiempo que lo requieran- y número de árboles o trozas $\left(\mathrm{m}^{3}\right)$, para una evaluación de gestión posterior; ii) análisis de los resultados; debido a que la versión actual solo los hace para cada día y acumula las bases de datos, se hace necesario el análisis de registros diarios en conjunto con la producción de la máquina. Esta última se lleva en registros separados.

\section{CONCLUSIONES}

El avance de la tecnología de teléfonos inteligentes ha proporcionado funciones más allá de las de un teléfono común. Los teléfonos actuales poseen una gran capacidad de almacenamiento, conectividad y -lo más importantede cómputo, una de las necesidades inmediatas de los ingenieros forestales en funciones de campo. Con la ayuda de ForestTime, los ingenieros forestales podrán evitar la molestia de llevar y operar varias herramientas y aun así adquirir los datos necesarios para estudios de tiempo y rendimiento de forma adecuada. Además, la variedad de registros puede estar relacionada de manera eficiente. La puesta en práctica de ForestTime demuestra que, con el software adecuado, los teléfonos inteligentes pueden traer a los ingenieros forestales y a otros profesionales grandes beneficios que van desde tareas múltiples y registro digital de datos para una gestión eficaz de la información que permite una recuperación sencilla de archivos y de la información almacenada.

\section{REFERENCIAS}

Ambrosio Y, E Tolosana. 2007. El control de tiempos y rendimientos en los trabajos forestales. El programa Kronos. Montes 87: 14-20.

Björheden R, MA Thompson. 1995. An international nomenclature for forest work study. In IUFRO 1995 20th World Congress. Subject Area S3.04, Tampere, Finland. Proceedings. p. 190-215. 
Boehm BW. 1988. A spiral model of software development and enhancement. Computer 21(5): 61-72.

Dianyuan H, W Chengduan. 2011. Tree height measurement based on image processing embedded in smart mobile phone. In International Conference on Multimedia Technology (ICMT), 26-28 July 2011. Proceedings. p. 3293-3296.

Dianyuan H, D Hui. 2011. The study on standing tree measurement based on image processing embedded in a smartphone. In International Conference on Image Analysis and Signal Processing (IASP), 21-23 Oct. 2011. Proceedings. p. 252-256.

Durán-Palma F, D López. 2009. Contract labour mobilisation in Chile's copper mining and forestry sectors. Employee Relations 31(3): 245-263.

García R. 2011. Sistema de guiado para incendios forestales. Tesis Bachelor. Castelldefels. Escola d'Enginyeria de Telecomunicació i Aeroespacial de Castelldefels, Universitat Politècnica de Catalunya. 86 p.

Itoh T, J Eizawa, N Yano, K Matsue, K Naito. 2010. Development of software to measure tree heights on the smartphone. Nihon Ringakkai Shi/Journal of the Japanese Forestry Society 92(4): 221-225.

Jeong IK, Y Ryu, MG Hong, C Kim. 2011. Simple application of Android-based smart phone for forest field survey. In 32nd Asian Conference on Remote Sensing 2011 (ACRS 2011),
3-7 October 2011, Taipei, Taiwan. Proceedings. p. 2280-2284. Lignum. 2013. Cosecha forestal de Pino radiata y Eucalyptus: negocio de valor y volumen. Lignum 23(142): 4-10.

Menard T, J Miller, M Nowak, D Norris. 2011. Comparing the GPS capabilities of the Samsung Galaxy S, Motorola Droid $\mathrm{X}$, and the Apple iPhone for vehicle tracking using FreeSim-Mobile. Proceedings. p. 985-990.

Monsen L. 2012. What is a mobile app and why do I want one? Consultado 04 de may. 2013. Disponible en http://iphoneapps.toptenreviews.com/what-is-a-mobile-app-and-whydo-i-want-one-.html.

Nelson R. 2012. Evaluating cellular evolution. EE: Evaluation Engineering 51(3): 14-20.

Pasicott P, GE Murphy. 2013. Effect of work schedule design on productivity of mechanised harvesting operations in Chile. New Zealand Journal of Forestry Science 43(1): 1-10.

Rolew AM. 1988. Siwork 3, version 1.1. Work study and field data collection system based on Husky Hunter handheld computer. Lyngby, Denmark. Danish Forest and Landscape Research Institute. 35 p.

Van Daele P. 2000. Work study in forestry. In Owen DL ed. South African Forestry Handbook. Pretoria, South Africa. South African Institute of Forestry. p. 407-413.

Wong W. 2011. Expect innovation from your new tablet. Electronic Design 59(15).

Recibido: 18.10 .12

Aceptado: 14.09.13 